\title{
MORPHOLOGY OF THE QUITINIZED STRUCTURES RELATED TO THE SPERMATHECA OF MUSCIDAE (INSECTA, DIPTERA)
}

\author{
Márcia Souto Couri ${ }^{1}$
}

\begin{abstract}
Little is known about the morphology of the quitinized structures related to the spermatheca of Muscidae (Diptera). Most papers illustrate only the spermatheca, which shape has little systematic significance when analyzed as a separate character. The analysis of these structures in 16 Muscidae species, made it possible to clarify and understand some characters, which can be used in further phylogenetic analysis. The species selected to this study belong to different sub-families: Muscinae, Muscini: Biopyrellia bipuncta (Wiedemann, 1830); Musca domestica Linnaeus, 1758; Azeliinae, Reinwardtini: Philornis univitattus Dodge, 1968; Cyrtoneurininae: Charadrella malacophaga Lopes, 1938; Cyrtoneurina polystigma (Wulp, 1896); Neomuscina neosimilis Snyder, 1949; Mydaeinae: Brontaea debilis (Williston, 1896); Graphomyia mexicana Giglio-Tos, 1893; Mydaea plaumani Snyder, 1941; Scutellomusca marginata (Albuquerque, 1954); Phaoninae: Dolichophaonia gallicola (ALbuquerque, 1958); Phaonia nigriventris (Albuquerque, 1954); Coenosiinae, Limnophorini: Limnophora saeva (Wiedemann, 1830); Coenosiini: Coenosia camorinensis Albuquerque, 1956 and Neodexiopsis paulistensis Albuquerque, 1956. The structures were described based on seven diagnostic characters.

KEY WORDS. Muscidae, morphology, spermatheca
\end{abstract}

The quitinized structures related to the spermatheca of Muscidae (Diptera) are nearly unknown. The shape of the spermatheca is commonly mentioned in the taxonomic papers, but it has little systematic significance when analyzed as a separate character.

The spermatheca and the allied structures have been studied by many authors in other dipteran families. ARTIGAS (1971) studied them in Asilidae, and had discussed their systematic value, concluding that they are of great value to define generic groups. He described the methodology of the dissection, and he also proposed a nomenclature for these structures. After this publication, many others were made in Asilidae, using diagnostic characters of the spermatheca and the allied structures (e.g. ARTIGAS et al. 1988, 1991; ARTIGAS \& PAPAVERO 1990).

Besides Asilidae, the spermatheca has been studied from a taxonomic point of view in other families as Mydidae (ARTIGAS 1990), Panthophtalmidae and Xylophagidae sensu lato (NAGATOMI \& LIU 1995), among others.

PAPE (1992) in a study on the phylogeny of Tachinidae family group, where Muscidae and Anthomyiidae were used as out-groups, dissected and analyzed the spermatheca of dipteran species belonging to the families Rhinophoridae, Sarcophagidae, Tachinidae, Calliphoridae, Oestridae and one Muscidae - Acanthiptera

1) Departamento de Entomologia, Museu Nacional. Quinta da Boa Vista, São Crsitóvão, 20940-040 Rio de Janeiro, Rio de Janeiro, Brazil. Fellow of the CNPq.

Revta bras. Zool. 15 (3): 597 - 603, 1998 
rohrelliformis (Acanthipterinae). Two characters related with the spermatheca were used in his analysis: the configuration of the spermathecal ducts relative to uterus and the relative capsule size. Concerning to the first character, PAPE (op. cit.) noticed that in Muscidae, two ducts are fused just before the uterus. This condition was considered in his analysis as plesiomorphic, while three separate ducts entering the uterous was the apomorphic state.

The purpose of this paper is to clarify the morphology of the allied structures of the spermatheca in Muscidae and to contribute to further phylogenetic analysis, through the use the new characters.

\section{MATERIAL AND METHODS}

I followed the methodology described in ARTIGAS (1971). Mainly due to the fragility of the allied structures, specially the spermathecal ducts it was necessary, in many cases, to dissect more than one exemplar of the same species. The spermathecal ducts can be very long, and in these cases, they were stretched out in order to see their openings. In Asilidae, the genital fork is easily observed, as it is strongly quitinized. In Muscidae, the only structures that can be seen are the spermatheca and the beginning of the spermathecal ducts, what demands a very careful dissection.

The species studied were: Muscinae, Muscini: Biopyrellia bipuncta (Wiedemann, 1830); Musca domestica Linnaeus, 1758; Azeliinae, Reinwardtini: Philornis univitattus Dodge, 1968; Cyrtoneurininae: Charadrella malacophaga Lopes, 1938; Cyrtoneurina polystigma (Wulp, 1896); Neomuscina neosimilis Snyder, 1949; Mydaeinae: Brontaea debilis (Williston, 1896); Graphomyia mexicana Giglio-Tos, 1893; Mydaea plaumani Snyder, 1941; Scutellomusca marginata (Albuquerque, 1954); Phaoninae: Dolichophaonia gallicola (ALbuquerque, 1958); Phaonia nigriventris (Albuquerque, 1954); Coenosiinae, Limnophorini:Limnophora saeva (Wiedemann, 1830); Coenosiini: Coenosia camorinensis Albuquerque, 1956 and Neodexiopsis paulistensis Albuquerque, 1956.

The terminology used by the authors is not uniform. ARTIGAS (1971), NAGATOMI \& LIU (1995), among others, used, for instance, the term "spermatheca" to all "complex", i.e. the spermatheca and the allied structures. The spermatheca was called by them "capsule" and, therefore, the spermathecal ducts were the "capsular ducts". MCALPINE (1981) restricted the term spermatheca for the receptacles, and the ducts are the "spermathecal ducts". This concept was used by PAPE (1992) and also is used here.

All studied material belongs to the collection of Museu Nacional, Rio de Janeiro.

\section{DESCRIPTIONS}

General plan. The quitinized structures of Muscidae spermatheca are very delicate, specially the spermathecal ducts. Three spermathecas are found in the ground plan. Two spermathecal glands are present (Fig. 1), opening close to the spermathecal ducts openings. They were not illustrated to all species. Two of the 
spermathecal ducts are fused just before entering the uterous. The valve, when seen, was located near the basal third of the spermathecal ducts.

The descriptions of the quitinized structures of the dissected species are presented below:

Biopyrellia bipuncta (Wiedemann, 1830): (Fig. 1). Spermatheca: elongate, equal sized, surface very corrugate, many pores near base and few pores at apex. Spermathecal ducts: medium sized, all similar in length.

Brontaea debilis (Williston, 1896): (Fig. 2) Spermatheca: elongate, equal sized, surface very corrugate, few pores near base. Spermathecal ducts: medium sized, all similar in length.

Charadrella malacophaga Lopes, 1938: (Fig. 3) Spermatheca: pear-shaped, equal sized, surface very corrugate, many pores near base. Spermathecal ducts: long, all similar in length.

Coenosia camorinensis Albuquerque, 1956: (Fig. 4) Spermatheca: pear shaped, equal sized, surface almost smooth, few pores near base. Spermathecal ducts: very long, all similar in length.

Cyrtoneurina polystigma (Wulp, 1896): (Fig. 5). Spermatheca: elongate, equal sized, surface very corrugate, many pores near base. Spermathecal ducts: long, all similar in length.

Dolichophaonia brasiliensis (Albuquerque, 1958): (Fig. 6). Spermatheca: pear shaped, equal sized, surface very corrugate, with a particular pattern, no pores seen. Spermathecal ducts: long, one longer than the others.

Dolichophaonia gallicola (Albuquerque, 1958): (Fig. 7). Spermatheca: pear shaped, equal sized, surface little corrugate, pores not seen. Spermathecal ducts: very long, one longer than the others.

Graphomyia mexicana Giglio-Tos, 1893: (Fig. 8). Spermatheca: round to elongate, equal sized, surface very corrugate, few pores near base. Spermathecal ducts: long, one longer than the others.

Limnophora saeva (Wiedemann, 1830): (Fig. 9). Spermatheca: round, equal sized, surface little corrugate, few pores scattered. Spermathecal ducts: long, all similar in length.

Musca domestica Linnaeus, 1758: (Fig. 10). Spermatheca: 2 elongate and one round and reduced, surface not much corrugate, many pores near base. Spermathecal ducts: short, all similar in length.

Mydaea plaumani Snyder, 1941: (Fig. 11). Spermatheca: round to elongate, equal sized, surface very corrugate, few pores near base. Spermathecal ducts: long, all similar in length.

Neomuscina neosimilis Snyder, 1949: (Fig. 12). Spermatheca: elongate, equal sized, surface very corrugate, few pores near base. Spermathecal ducts: not very long, all similar in length.

Neodexiopsis paulistensis Albuquerque, 1956: (Fig. 13). Spermatheca: round, equal sized, surface almost smooth, many pores near base. Spermathecal ducts: very long, all similar in length.

Phaonia nigriventris (Albuquerque, 1954): (Fig. 14). Spermatheca: pear shaped, equal sized, surface very corrugate, many pores near base. Spermathecal ducts: very long, all similar in length. 
Philornis univitattus Dodge, 1968: (Fig. 15). Spermatheca: round, equal sized, surface very corrugate, few pores scattered. Spermathecal ducts: long, one longer than the others.

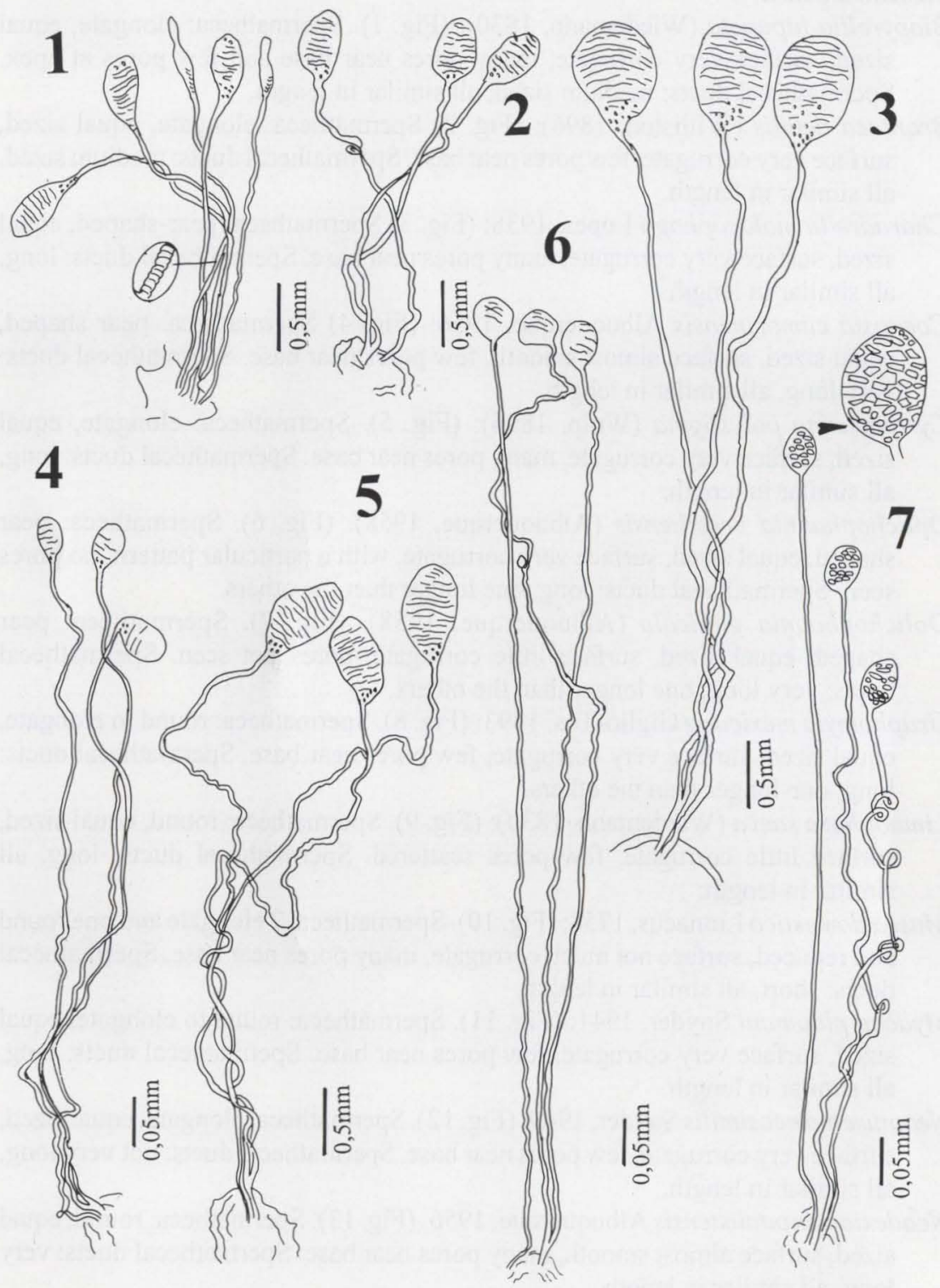

Figs 1-7. Quitinized structures of spermatheca. (1) Biopyrellia bipuncta; (2) Brontaea debilis; (3) Charadrella malacophaga; (4) Coenosia camorinensis; (5) Cyrtoneurina polystigma; (6) Dolichophaonia brasiliensis; (7) Dolichophaonia gallicola. 
Scutellomusca marginata (Albuquerque, 1954): (Fig. 16). Spermatheca: elongate, equal sized, surface very corrugate, few pores near base. Spermathecal ducts: medium sized, one longer than the others.

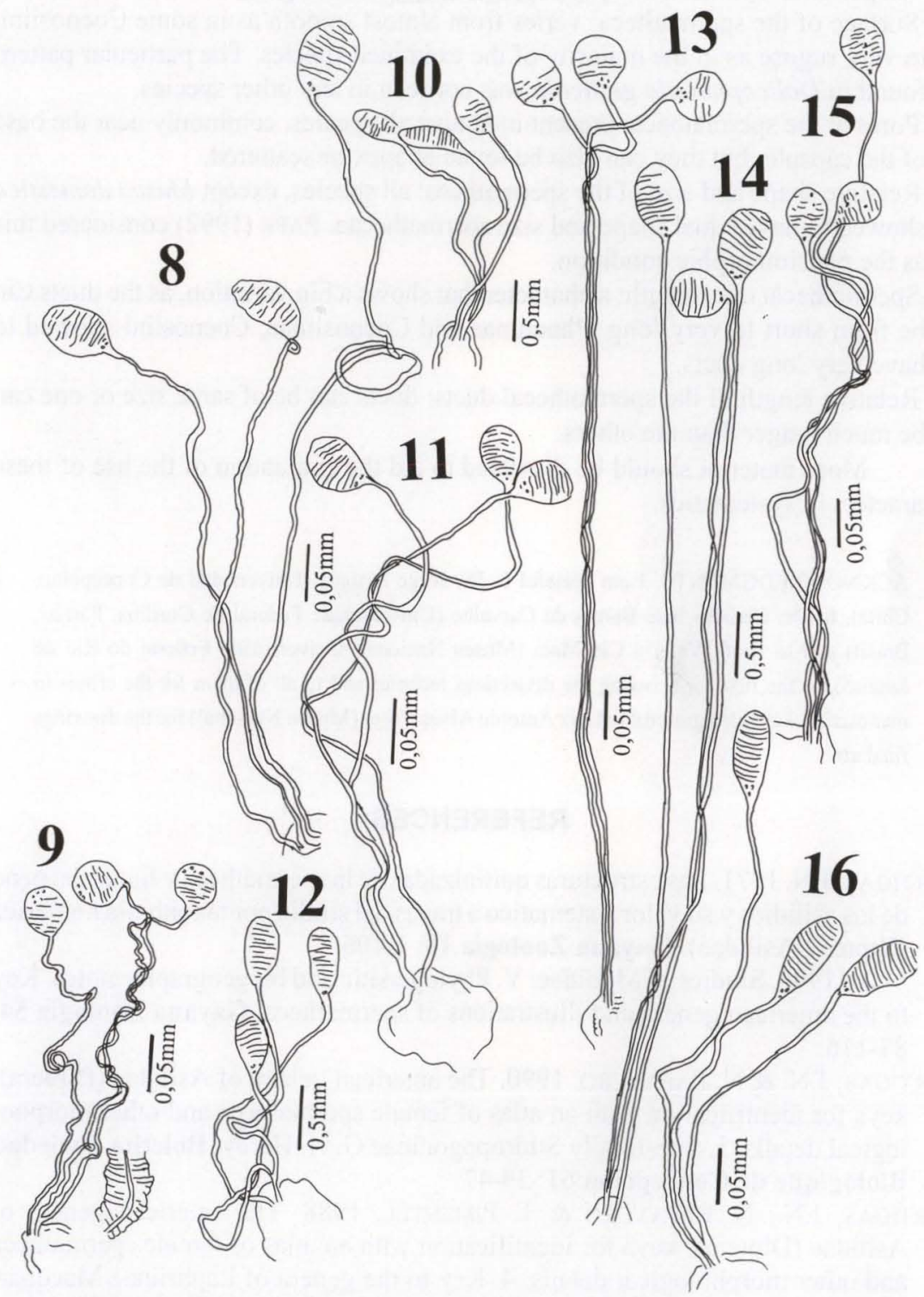

Figs 8-16. Quitinized structures of spermatheca. (8) Graphomyia mexicana; (9) Limnophora saeva; (10) Musca domestica; 1758; (11) Mydaea plaumani; (12) Neomuscina neosimilis; (13) Neodexiopsis paulistensis; (14) Phaonia nigriventris; (15) Philornis univitattus; (16) Scutellomusca marginata. 


\section{DISCUSSION}

The list below resumes the diagnostic characters and their states:

1) Shape of the spermatheca: round, pear-shaped or elongate

2) Surface of the spermatheca: varies from almost smooth as in some Coenosiini, to very rugose as in the majority of the examined species. The particular pattern found in Dolicophaonia gallicola was not seen in any other species.

3) Pores at the spermatheca: present in almost all species, commonly near the base of the capsule; but they can also be found at apex or scattered.

4) Relative shape and size of the spermatheca: all species, except Musca domestica showed to have equal shape and size spermathecae. PAPE (1992) considered this as the pleisiomorphic condition.

5) Spermathecal duct length: a character that shows a big variation, as the ducts can be from short to very long. Phaoninae and Coenosiinae, Coenosiini showed to have very long ducts.

6) Relative length of the spermathecal ducts: ducts can be of same size or one can be much longer than the others.

More material should be dissected to aid the evaluation of the use of these characters in systematics.

ACKNOWLEDGMENTS. I am grateful to Dr. Jorge Artigas (Universidad de Concepcion, Chile), to Dr. Claudio José Barros de Carvalho (Universidade Federal de Curitiba, Paraná, Brasil) and to Profa Valeria Cid Maia (Museu Nacional, Universidade Federal do Rio de Janeiro), to the first for showing the dissections technics and to all of them for the critics in manuscript. I am also grateful to Luiz Antonio Alves Costa (Museu Nacional) for the drawings final art.

\section{REFERENCES}

ARTIGAS, J.N. 1971. Las estructuras quitinizadas de la spermatheca y funda del pene de los asilidios y su valor sistematico a traves del studio por taxonomia numerica (Diptera, Asilidae). Gayana Zoologia 18: 1-106.

. 1990. Studies of Mydidae. V. Phylogenetic and biogeographic notes. Key to the american genera and illustrations of spermatheca. Gayana Zoologia 54: 87-116.

Artigas, J.N. \& N. PAPAVERo. 1990. The american genera of Asilidae (Diptera): keys for identification with an atlas of female spermatheca and other morphological details. 5. Sub-family Stidropogoninae G. H. Hardy. Boletim Sociedad Biologique de Concepcion 61: 39-47.

Artigas, J.N.; N. PAPAVERo \& T. Pimentel. 1988. The american genera of Asilidae (Diptera): keys for identification with an atlas of female spermatheca and other morphological details. 4. Key to the genera of Laphriinae Macquart (except tribe Atomosiini Hermann), with the descriptions of three new tribes and five new species. Boletim do Museu Paraense Emílio Goeldi, nova série Zoologia, 4 (2): 211-256.

Artigas, J.N.; N. Papavero \& A. L. Serra. 1991. The american genera of 
Asilidae (Diptera): keys for identification with an atlas of female spermatheca and other morphological details. 6. Tribe Atomosiini and a catalogue of the neotropical species. Gayana Zoologia 55 (1): 53-85.

MCALPINE, J.F. 1981. Morphology and terminology - adults, p.9-63. In: J.F. MCAlPINE; B.V. PeTERSON; G.E. SHEWEll; H.J. TESKEY; J.R. VOCKEROTH \& D.M. Wood (Eds). Manual of Neartic Diptera. Research Branch, Agriculture Canada, Monograph No. 27, Vol. 1, 1-647.

NAGATOMI, A. \& N. LIU. 1995. Spermatheca and female terminalia of Pantophthalmidae and Xylophagidae s.lat. (Diptera). Annals of the Entomological Society of America 88 (5): 604-623.

PAPE, T. 1992. Phylogeny of the Tachinidae family-group (Diptera:Calyptratae). Tijdschrift voor Entomologie 135: 43-86.

Recebido em 27.III.1997; aceito em 13.VII.1998. 